\title{
Seroprevalence of Bordetella pertussis among vaccinated and unvaccinated pregnant women and newborn infants in a university hospital of Buenos Aires
}

\author{
Juan J. Bosch, M.D. ${ }^{a}$, Hilaria Fernández, M.D. ${ }^{a}$, Fernando P. Polak, M.D. ${ }^{b}$, \\ Gabriel Musante, M.D. ${ }^{a}$, Romina Libster, M.D. ${ }^{a}$ and Manuel Rocca Rivarola, M.D. ${ }^{a}$
}

\begin{abstract}
Introduction. Pertussis is a highly contagious disease caused by Bordetella pertussis. It poses a high morbidity and mortality rate, especially among infants younger than 6 months old. In Argentina, pertussis incidence and mortality have increased over the past three decades. Objective. To establish Bordetella pertussis antibody titers among pregnant women in their third trimester and among newborn infants, as measured in cord blood.

Methods. This was an observational, crosssectional study. The study started in 2011; at that time, pertussis vaccination was not mandatory for pregnant women as per the national immunization schedule, only optional. Maternal antibodies were measured in the last trimester of pregnancy for women and in cord blood for newborn infants. Antibody titers were determined using Abcam's anti-Bordetella pertussis toxin (PT) IgG in vitro ELISA kit. The $\chi^{2}$ test was used to compare prevalence rates. Results. The study included 111 mother-newborn infant dyads; 35 infants from unvaccinated mothers (before the introduction of the vaccine) and 76 from vaccinated mothers. Positive IgG antibodies were found in $92 \%$ (70/76) of infants born from vaccinated mothers whereas $100 \%$ $(35 / 35)$ of infants born from unvaccinated mothers had negative results for antibodies; $p<0.001$.

Conclusion. In the vaccinated population of this study, $92 \%$ of infants had positive IgGantibodies. This study supports the need for maternal immunization against Bordetella pertussis to provide protection to newborn infants.

Key words: pertussis, pertussis toxin, antibodies, maternal immunization.
\end{abstract}

http:/ / dx.doi.org/10.5546/ aap.2017.eng.311

Funding:

The study was

conducted with the support of the Florencio Fiorini Medical Research Stimulation Fellowship for year 2013.

Conflict of interest: None.

Received: 10-15-2016 Accepted: 1-4-2017

\section{INTRODUCTION}

Pertussis or whooping cough is a highly contagious disease caused by Bordetella pertussis. Its morbidity and mortality rate is high, especially among partially immunized individuals, and newborn infants are the highest-risk group. ${ }^{1}$

In Argentina, the incidence of pertussis decreased since the introduction of the triple bacterial vaccine in the 1970s (triple bacterial: tetanus, diphtheria, and pertussis) until 2003 (639 cases; 1.8/100 000 inhabitants). Between 2003 and 2005, the number of cases increased to 2060, with a 5.7/100 000 inhabitants reporting rate. ${ }^{2}$

The increase in the incidence of Bordetella pertussis among adolescents and young adults has turned them into reservoirs and potential vectors of pertussis among less protected risk groups, e.g. newborn infants, whose morbidity and mortality rate is much higher.

In 2009, the national immunization schedule introduced the triple bacterial vaccine against diphtheria, tetanus, and pertussis (Tdap vaccine) at 11 years old, and subsequently recommended it for pregnant women as of 20 weeks of gestation. It was first part of a campaign, which started in February 2012, and was then made mandatory as per the national immunization schedule in January $2014 .^{3}$

At present, it is indicated with each pregnancy and even for postpartum women who did not receive the vaccine during pregnancy, health care staff caring for infants younger 
than 1 year old, and people living with preterm infants with a birth weight $<1500$ grams. ${ }^{4}$

Data have been recently published by the National Division for the Control of VaccinePreventable Diseases (Dirección Nacional de Control de Enfermedades Inmunoprevenibles, DiNaCEI) of the National Ministry of Health of Argentina: maternal coverage provided by the Tdap vaccine was 50.9\% during the first campaign year (2012), and reached $67.2 \%$ in 2013. Pertussis incidence reduced by $60 \%$ between 2011 and 2013, from 2821 reported cases in 2011 to 1117 in 2013. ${ }^{5}$

Among those with Bordetella pertussis infection, $90 \%$ are infants younger than 1 year old $(75 \%$ are younger than 6 months old); ${ }^{6} 50 \%$ require hospitalization, and the mortality rate ranges between 6\% and 9\%. ${ }^{7}$ In Argentina, as in the Netherlands and the United States, pertussis incidence and mortality have increased over the past three decades. Failure to induce long-term immunity with present vaccines and antigenic divergence between local strains and vaccines may worsen the impact of such decreasing immunity. ${ }^{8}$

Our hypothesis proposed that the presence of maternal antibodies was related to the presence of antibodies in newborn infants. The objective of this study was to establish the presence of antibodies against Bordetella pertussis among pregnant women in their third trimester and among newborn infants in cord blood in relation to maternal immunization.

\section{POPULATION AND METHODS}

An observational, cross-sectional study was conducted between August 2011 and May 2014 at Hospital Universitario Austral.

When the study started, the pertussis vaccine was not mandatory for pregnant women. In February 2012, the National Ministry of Health started an immunization campaign among pregnant women, and the vaccine was finally included in the national immunization schedule in January 2014.

This hospital caters for a large population from the North of Greater Buenos Aires and is the second most important maternity center in the district of Pilar. Approximately 2000 infants are born here each year; $4 \%$ of patients followed at this hospital are born in different hospitals.

All pregnant women seen at the hospital were invited to participate in the study using a written informed consent form assessed and approved by the Institutional Review Board of
Hospital Universitario Austral and the Central Ethics Committee of the Ministry of Health of the Province of Buenos Aires (file no $2919 / 269 / 11$ ).

\section{Inclusion criteria}

Pregnant women older than 18 years old, who had given their written informed consent, had a blood sample collected during their last trimester prenatal checkup at the hospital and would have birth at this facility were included.

Vigorous newborn infants who had a cord blood sample collected large enough to measure the presence of anti-pertussis toxin antibodies were included.

\section{Exclusion criteria}

Newborn infants whose Apgar score indicated they were not vigorous or whose status prevented sample collection were excluded, as well as mothers with a twin pregnancy, who were not able to give their consent, who were younger than 18 years old at the time of consent signature, and who had not had a blood sample collected during their last trimester prenatal checkup.

Mothers who were not followed-up at the hospital or who gave birth at a different facility were excluded from the analysis.

\section{Study procedures}

Pregnant women had a blood sample collected during their last trimester prenatal checkup, and a $500 \mu \mathrm{lt}$ serum aliquot was obtained from this blood sample and stored. Samples were anonymized and identified with an alphanumeric code.

Newborn infants had a cord blood sample collected for the determination of ABO blood group and Rhesus factor, and a $500 \mu$ lt aliquot was obtained from this sample after making the determination. This sample was labeled and matched to the corresponding maternal sample.

Samples were stored in a refrigerator at $-70{ }^{\circ} \mathrm{C}$ in the Hospital Austral laboratory.

Antibody titers were measured simultaneously in all samples once the study was completed. An Abcam's anti-Bordetella pertussis toxin (PT) IgG in vitro ELISA kit was used.

The following maternal outcome measures were assessed: age, medical history, antiphospholipid syndrome, smoking, vaccination status. Pregnancy-related outcome measures included pregnancy history, such as hypertension, gestational diabetes, hypothyroidism, corticosteroid use during pregnancy, smoking 
or alcohol use during pregnancy, number of antenatal care visits, pregnancy number, delivery mode (vaginal or C-section), infections or other diseases during pregnancy that may impact the placental-fetal unit.

Among newborn infants, outcome measures included sex, gestational age, birth weight, and Apgar score at 1 and 5 minutes.

The $\chi^{2}$ test was used to compare prevalence rates, and a $p$ value $<0.05$ was considered significant. Statistical analysis was done using the Epi Info $7^{\circledR}$ software.

\section{RESULTS}

During the protocol period, 3398 infants were born at the hospital; 2883 met the eligibility criteria for the study. Out of the 215 mothers who signed the informed consent, a blood sample was obtained from 181 during their third trimester. Of these, 59 did not give birth at this hospital; also, it was not possible to obtain the cord blood sample from 11 of the infants born here for different reasons (6 were not vigorous as per their Apgar score, and the sample volume was not enough in 5). Finally, 111 mother-newborn infant dyads were included; 35 infants born from unvaccinated mothers and 76 born from women vaccinated during their second trimester of pregnancy.

The average age of enrolled mothers was 30 years old, and there were no differences between groups. Only one mother was between 18 and 20 years; 51 were between 20 and 30 years; 43, between 31 and 35 years; and 16, older than 35 years. Besides, $80 \%$ of mothers had completed secondary education; $10 \%$ were in secondary school; and $10 \%$ had completed primary education. Also, $90 \%$ had completed more than 5 antenatal care visits; and 10\%, more than 3 .

The average gestational age at birth was 39 weeks; 3 infants were born at 37 weeks; 19 , at 38 weeks; 68, at 39 weeks; 16 , at 40 weeks; and 5 , at 41 weeks. The average birth weight was $3.475 \mathrm{~kg} ; 7$ infants had a birth weight between 2 and $2.5 \mathrm{~kg}$; 58 , between 2.5 and $3.5 \mathrm{~kg}$; and $46,>3.5 \mathrm{~kg}$ (see Table 1). The Apgar score at 1 minute was 7 in 2 patients born with nuchal cord entanglements and $>8$ at 5 minutes in all patients.

Positive IgG antibodies were found in $92 \%$ $(70 / 76)$ of infants born from vaccinated mothers whereas $100 \%$ (35/35) of infants born from unvaccinated mothers had negative results for antibodies; $p<0.001$. Among vaccinated mothers, $8 \%$ of infants did not have anti-pertussis antibodies. None of these 6 mothers had a significant medical history.

No differences were observed among the studied outcome measures and the presence or absence of antibodies, except for the history of immunization.

\section{DISCUSSION}

Multiple studies have been conducted to assess the presence of protective antibodies against Bordetella pertussis among mothers and the cord blood of their infants. ${ }^{9,10}$

In our study, infants born from unvaccinated mothers were negative for cord blood antibodies, and most vaccinated mothers gave birth to infants positive for cord blood antibodies.

In a study conducted by Nooitgedagt et al., mothers with low antibody titers transferred a lower antibody level to the fetus, leaving their newborn infant at a higher risk for disease in the first months of life. ${ }^{11}$

In a study conducted by Fallo in Argentina to establish pertussis seroprevalence among young adults, women in their immediate post-partum period, and umbilical cord blood, total antibodies against whole-cell pertussis were measured. It was observed that both mothers and umbilical cords had low antibody titers, which evidenced lower antibody titers among 25-39 year-old women. ${ }^{12}$

TABLE 1. Demographic characteristics and main outcome measures assessed in the studied population

\begin{tabular}{lcc}
\hline \multicolumn{3}{c}{ Mothers (n: 111) } \\
\hline Vaccinated & Unvaccinated \\
\hline Age $\bar{x}$ & $76 / 68.5 \%$ & $35 / 31.5 \%$ \\
$\%$ of C-sections & 29.9 & 30 \\
$\%$ who smoke & $38 \%$ & $39 \%$ \\
$\%$ of GHTN & $6.30 \%$ & $5.80 \%$ \\
$\%$ of hypothyroidism & $5.00 \%$ & $5.10 \%$ \\
GDBT & $9.60 \%$ & $9.00 \%$ \\
APS & $11.53 \%$ & $10.90 \%$ \\
UTI & $5.70 \%$ & $5.90 \%$ \\
Cholestasis & $5.10 \%$ & $5.10 \%$ \\
& $1.70 \%$ & $0.00 \%$ \\
\hline & Bebés (n: 111) \\
\hline GA $\bar{x}$ & Vaccinated & Unvaccinated \\
Weight $\bar{x}$ & 39.36 & 39 \\
$\%$ of boys & 3420 & 3530 \\
\hline
\end{tabular}

GHTN: gestational hypertension; GDBT: gestational diabetes; APS: antiphospholipid syndrome;

UTI: urinary tract infection; GA: gestational age. 
The titer of transplacental anti-pertussis antibodies decreases in infants with a 6-week half-life and, by 6 months old, infants have no detectable anti-pertussis antibodies. Even with an effective transplacental passage, the infant's passive immunity is not enough. ${ }^{13-15}$

According to the national immunization schedule, the whole-cell pertussis vaccine is indicated at 2, 4, and 6 months old, with a booster dose at 18 months old, 6 and 11 years old, and then every 10 years. Individuals who receive only the acellular vaccine have a lower immunity because their antibody titers reduce by 4 years following vaccine administration. ${ }^{16}$ For this reason, as of this year, it was decided to introduce the triple acellular vaccine for pregnant women with each pregnancy.

In our study, the main outcome measure associated with the presence of cord blood antibodies was pregnant women immunization. The main factors related to transplacental antibody passage included gestational age, maternal infections, and maternal antibody titers. ${ }^{17}$

In a study conducted by Heininger, preterm newborn infants had lower anti-pertussis antibodies, and the transplacental passage range was higher among term newborn infants. ${ }^{15}$ Healy et al. observed that anti-pertussis toxin antibodies were lower among Hispanic teenage mothers. ${ }^{18}$ Although that study did not include a non-Hispanic population control group, findings are consistent with those of Plans et al., who evidenced that the presence of antibodies reduced with age. ${ }^{19}$ In turn, infants born from teenage mothers had a higher risk for pertussis. ${ }^{20}$

In our study, no preterm infants were included, and maternal age was not related to the presence or absence of antibodies, although mothers younger than 18 years old were excluded and only one was younger than 20 years old.

The assessment of the maternal pertussis immunization program in England showed that protection was approximately $90 \%$ at 3 years of follow-up, which was sustained in spite of having changed from a triple acellular vaccine, as the one used in our study, to a pentavalent vaccine..$^{21,22}$

This study was done during routine interventions provided to pregnant women at our hospital, and cord blood samples were collected once the blood groups and Rhesus factor were determined. Such characteristic has certain limitations that are worth mentioning: although pregnant women were vaccinated in their second trimester, the precise date was unknown. This may be important to assess the $8 \%$ of mothers whose infants had negative antibodies because not enough time may have elapsed for the mother to develop vaccine-induced antibodies, and antibodies may not have been high at the time of transplacental transfer.

In February 2016, an update by the UK Joint Committee on Vaccination and Immunisation (JCVI) recommended that pregnant women should ideally receive the vaccine before 28 weeks of gestation to improve antibody transplacental passage to the fetus. ${ }^{23}$ Some studies recommend early immunization during the second trimester, with improved outcomes if done between 13 and 25 weeks of gestation. ${ }^{24}$

The antibody kit used in this study was qualitative and provided data on the presence or absence of antibodies but does not allow to measure geometric means to establish antibody passage levels.

\section{CONCLUSION}

In the vaccinated population of this study, 92\% of infants had positive IgG antibodies. This study supports the need for maternal immunization against Bordetella pertussis to provide protection to newborn infants.

\section{Acknowledgments}

We would like to thank the Department of Academic Development of Hospital Universitario Austral for their help with statistical data and analysis article editing; to Silvina Coviello, Biochemist, and Sandy Yoder, Biochemist, for their generous collaboration to start this study.

\section{REFERENCES}

1. Centers for Disease Control and Prevention (CDC). Pertussis--United States, 1997-2000. MMWR Morb Mortal Wkly Rep 2002;51(4):73-6.

2. Hozbor D, Mooi F, Flores D, Weltman G, et al. Pertussis epidemiology in Argentina: trends over 2004-2007. J Infect 2009;59(4):225-31.

3. Vizzotti C, Neyro S, Katz N, Juárez MV, et al. Maternal immunization in Argentina: A storyline from the prospective of a middle income country. Vaccine 2015;33(47):6413-9.

4. Dirección de Control de Enfermedades Inmunoprevenibles. pertussis. Buenos Aires: Ministerio de Salud de la Nación; 2015. [Accessed on: January $6^{\text {th }}, 2017$. Available at: http:// www.msal.gob.ar/dicei/index.php/ciudadanos/vacunasdel-calendario/353-tos-convulsa.

5. Vizzotti C, Juarez MV, Bergel E, Romanin V, et al. Impact of a maternal immunization program against pertussis in a developing country. Vaccine 2016;34(50):6223-8.

6. Gentile A. Infección por Bordetella pertussis. Arch Argent Pediatr 2010;108(1):78-81.

7. Broder KR, Cortese MM, Iskander JK, Kretsinger K, et al. Preventing tetanus, diphtheria, and pertussis among 
adolescents: use of tetanus toxoid, reduced diphtheria toxoid and acellular pertussis vaccines recommendations of the Advisory Committee on Immunization Practices (ACIP). MMWR Recomm Rep 2006;55(RR-3):1-34.

8. Mooi FR, de Greeff SC. The case for maternal vaccination against pertussis. Lancet Infect Dis 2007;7(9):614-24.

9. Healy CM, Munoz FM, Rench MA, Halasa NB, et al. Prevalence of pertussis antibodies in maternal delivery, cord, and infant serum. J Infect Dis 2004;190(2):335-40.

10. Gonik B, Puder KS, Gonik N, Kruger M. Seroprevalence of Bordetellapertussis antibodies in mothers and their newborn infants. Infect Dis Obstet Gynecol 2005;13(2):59-61.

11. Nooitgedagt JE, de Greeff SC, Elvers BH, de Melker HE, et al. Seroprevalence of Bordetella pertussis infection during pregnancy measured by $\operatorname{IgG}$ antibodies against pertussis toxin. Clin Infect Dis 2009;49(7):1086-9.

12. Fallo A, Manonelles G, Hozbor D, Lara C, et al. Estudio de seroprevalencia de coqueluche en adultosjóvenes, mujeres en puerperio inmediato y sangre del cordón umbilical. Arch Argent Pediatr 2014;112(4):315-22.

13. Edwards KM. Pertussis: an important target for maternal immunization. Vaccine 2003;21(24):3483-6.

14. Haberling DL, Holman RC, Paddock CD, Murphy TV. Infant and maternal risk factors for pertussis-related infant mortality in the United States, 1999 to 2004. Pediatr Infect Dis J 2009;28(3):194-8.

15. Heininger U, Riffelmann M, Leineweber B, Wirsing von Koenig $\mathrm{CH}$. Maternally derived antibodies against Bordetella pertussis antigens pertussis toxin and filamentous hemagglutinin in preterm and full term newborns. Pediatr Infect Dis J 2009;28(5):443-5.
16. Schwartz KL, Kwong JC, Deeks SL, Campitelli MA, et al. Effectiveness of pertussis vaccination and duration of immunity. CMAJ 2016;188(16):E399-E406.

17. Huang CC, Chen PM, KuoJK, Chiu WH, et al. Experimental Whooping cough. N Engl J Med 1962;266:105-11.

18. Healy CM, Rench MA, Edwards KM, Baker CJ. Pertussis serostatus among neonates born to Hispanic women. Clin Infect Dis 2006;42(10):1439-42.

19. Plans P, Jansà J, Doshi N, Harrison TG, et al. Prevalence of pertussis antibodies in umbilical cord blood samples in Catalonia, Spain. Pediatr Infect Dis J 2008;27(11):1023-5.

20. Izurieta HS, Kenyon TA, Strebel PM, Baughman AL, et al. Risk factors for pertussis in young infants during an outbreak in Chicago in 1993. Clin Infect Dis 1996;22(3): 503-7.

21. Amirthalingam G, Andrews N, Campbell H, RibeiroS, etal. Effectiveness of maternal pertussis vaccination in England: an observational study. Lancet 2014;384(9953):1521-8.

22. Amirthalingam G, Campbell H, Ribeiro S, Fry NK, et al. Sustained effectiveness of the maternal pertussis immunization program in England 3 years following introduction. Clin Infect Dis 2016;63(suppl 4):S236-43.

23. Joint Committee on Vaccination and Immunisation. Minute of the meeting on 3 February 2016. [Accessed on: January $6^{\text {th }}, 2017$. Available at: https: / / app.box.com/s / iddfb4pp wkmtjusir2tc/1/2199012147/66698939189/1.

24. Eberhardt CS, Blanchard-Rohner G, Lemaître B, Boukrid $M$, et al. Maternal immunization earlier in pregnancy maximizes antibody transfer and expected infant seropositivity against pertussis. Clin Infect Dis 2016;62(7):829-36. 